'Departamento de Psiquiatría, Facultad de Medicina, Pontificia Universidad Católica de Chile. Santiago, Chile.

${ }^{2}$ Departamento de Gastronutrición infantil, Facultad de Medicina, Pontificia Universidad Católica de Chile. Santiago, Chile.

${ }^{3}$ Unidad de Trastornos de la Conducta Alimentaria, Pontificia Universidad Católica de Chile. Santiago, Chile.

Este trabajo no contó con financiamiento externo. Conflictos de interés: Ninguno.

Recibido el 11 de diciembre de 2015, aceptado el 7 de septiembre de 2016 .

Correspondencia a: Dra. Melina Voge Jefa Unidad de Trastornos de la Conducta Alimentaria Pontificia Universidad Católica de Chile. Santiago, Chile. melina.vogel@gmail.com

\section{Actualizaciones en el manejo intrahospitalario de la anorexia nerviosa: recomendaciones prácticas}

\author{
NICOLE VÁSQUEZ ${ }^{1}$, PASCUALA URREJOLA ${ }^{2,3}$, MELINA VOGEL ${ }^{1,3}$
}

\section{An update on inpatient treatment of anorexia nervosa: practical recommendations}

Anorexia Nervosa (AN) is the psychiatric illness with the highest mortality, especially if it is associated with psychiatric and somatic comorbidity. Medical complications can be multiple and jeopardize the normal development of children and adolescents, even permanently. Although its prevalence is lower compared with other psychiatric disorders, its tendency to chronicity and the severity of its consequences are remarkable. Although outpatient treatment of anorexia is privileged as far as possible, the risks associated with poor response to treatment or lack of adherence of the patient or family, require the possibility of hospitalization at any time of the disease. We searched and analyzed the national and international literature available (especially clinical guidelines) about the indication for hospital treatment in AN and the interventions recognized as necessary and effective during hospitalization. Despite the lack of standardized criteria for hospitalization, the available information converge on the need for a multidisciplinary work by a specialist team, to make family interventions especially in adolescents and tailor treatment according to the individual physical, psychological and social needs.

(Rev Med Chile 2017; 145: 650-656)

Key words: Anorexia; Eating; Feeding and Eating Disorders; Inpatients.

\section{L} a anorexia (AN) es un trastorno psiquiátrico severo, con consecuencias físicas y psicológicas habitualmente devastadoras. Se caracteriza por bajo peso, restricción alimentaria e intenso temor a engordar, pudiendo asociarse también a conductas anormales para evitar subir de peso, como ejercicio físico extenuante, uso de laxantes o diuréticos, o la autoprovocación del vómito. Se presenta con mayor frecuencia en mujeres que en hombres ${ }^{1}$ y suele debutar en la adolescencia o adultez temprana ${ }^{2}$. Su prevalencia a nivel mundial es de $0,4 \%$, mientras que en Chile no se cuenta con cifras específicas para este trastorno ${ }^{3}$. Es una de las principales causas de AVISA en mujeres jóvenes y su costo por caso es al menos similar al de la esquizofrenia ${ }^{4}$. Por otra parte, se ha demostrado que su detección y tratamiento precoz mejora el pronóstico, disminuyendo su cronificación y sus complicaciones tanto médicas como psiquiátricas $^{5-7}$. A propósito de la importancia de su detección precoz y del impacto negativo de esta patología, tanto individual como social, la quinta edición del manual diagnóstico y estadístico de la Academia Americana de Psiquiatría ${ }^{3}$ incorpora cambios recientes en los criterios diagnósticos de la AN que permiten su pesquisa de manera menos excluyente (particularmente durante la adolescencia), especificando subtipos purgativo y restrictivo, y categorizando por gravedad según el índice de masa corporal (IMC) (Figura 1).

Aunque este trastorno presenta una baja prevalencia, sus consecuencias pueden ser tan 
A. Restricción de la ingesta calórica en relación a los requerimientos que lleva a un bajo peso significativo en el contexto de edad, género, momento del desarrollo y salud física. Bajo peso significativo se define como un peso menor al mínimo normal o, para niños y adolescentes, menor al mínimo esperado.

B. Miedo intenso a subir de peso o volverse gordo/a, o conducta persistente que interfiere con el aumento de peso, aún teniendo un bajo peso significativo.

C. Alteración en la manera que se experimenta la propia silueta o el propio peso, exagerada influencia del peso o de la forma corporal en la autoevaluación, o persistente falta de reconocimiento de la seriedad del bajo peso corporal actual.

Figura 1. Criterios diagnósticos DSM-5.

devastadoras y su tratamiento ser de tal complejidad, que algunos autores la sitúan como uno de los trastornos más serios de la psiquiatría ${ }^{8,9}$. Se le ha atribuido una tasa de mortalidad 6,5 veces mayor que la población general ${ }^{10}$, mientras que algunos autores la destacan como la enfermedad psiquiátrica con mayor riesgo de muerte ${ }^{9}$, siendo el suicidio responsable de 20 a $30 \%$ de su letalidad $^{8,9,11}$. Las complicaciones médicas de la desnutrición pueden ser múltiples y poner en riesgo el desarrollo normal del adolescente, incluso en forma permanente ${ }^{12}$.

A pesar que se privilegia su manejo ambulatorio, los riesgos asociados a una mala respuesta al tratamiento o a la falta de adherencia del paciente o su familia, requieren de la posibilidad de un abordaje intrahospitalario en cualquier momento de la enfermedad. Sin embargo, existe poco consenso respecto a cuáles son las estrategias más eficaces para el manejo dentro del hospital: los equipos médicos pueden enfrentarse con frecuencia a las graves dificultades de manejo de las complicaciones de la desnutrición y, a la vez, a los constantes sabotajes de los pacientes frente a la realimentación. Cabe destacar la difusión mediática de casos recientes de pacientes con $\mathrm{AN}$ que fueron tratados en unidades médicas intrahospitalarias, pero que, por falta de experiencia en su manejo, se deterioraron y fallecieron por problemas psiquiátricos y complicaciones médicas, como el síndrome de realimentación o una prescripción calórica inapropiadamente baja.

En virtud de lo anterior, este artículo busca revisar y sintetizar las recomendaciones disponibles, tanto a nivel nacional como internacional, que se refieran a la indicación de tratamiento intrahospitalario en la AN y a las intervenciones reconocidas como necesarias y eficaces durante la hospitalización.

\section{Material y Método}

Se realiza búsqueda en inglés y español, usando Pubmed, PsycInfo, Epistemonikos, Google Académico y Scielo, con los siguientes términos: anorexia nervosa, anorexia nerviosa, eating disorders, trastornos de la conducta alimentaria, trastornos de alimentación, inpatient, inpatient treatment, hospitalization, manejo intrahospitalario, tratamiento intrahospitalario. Se revisan estudios descriptivos, randomizados controlados, meta-análisis y guías clínicas sustentadas por la evidencia científica internacional entre los meses de mayo y julio de 2015. Las guías clínicas mostraron recomendaciones más concretas y sistematizadas respecto al tratamiento intrahospitalario de la $\mathrm{AN}$, por lo cual sólo fueron incluidas estas últimas en los resultados.

\section{Resultados}

La evidencia sobre el manejo intrahospitalario de pacientes con AN está en pleno desarrollo. Muchas de las recomendaciones se basan en opiniones de expertos, en ausencia de evidencia significativa de estudios controlados $\mathrm{u}$ otras investigaciones sistemáticas. La dificultad de generar estudios de alto nivel de evidencia se debe, entre otras cosas, al bajo número de casos, la falta de condiciones control válidas y el uso de diferentes aproximaciones terapéuticas en las cuales los elementos de tratamiento individuales no son descritos.

En el Anexo 1, se detallan los aspectos más relevantes de cada guía clínica por separado: 1) Descripción del equipo de trabajo y población objetivo; 2) Evaluación del riesgo (elementos clínicos -tanto médicos como psiquiátricos- que predicen un peor pronóstico a corto plazo); 3) Indicaciones de hospitalización; 4) Principales aspectos nutricionales, psiquiátricos y psicoterapéuticos del manejo intrahospitalario de estas pacientes. La Tabla 1 presenta un resumen de dichas recomendaciones. 


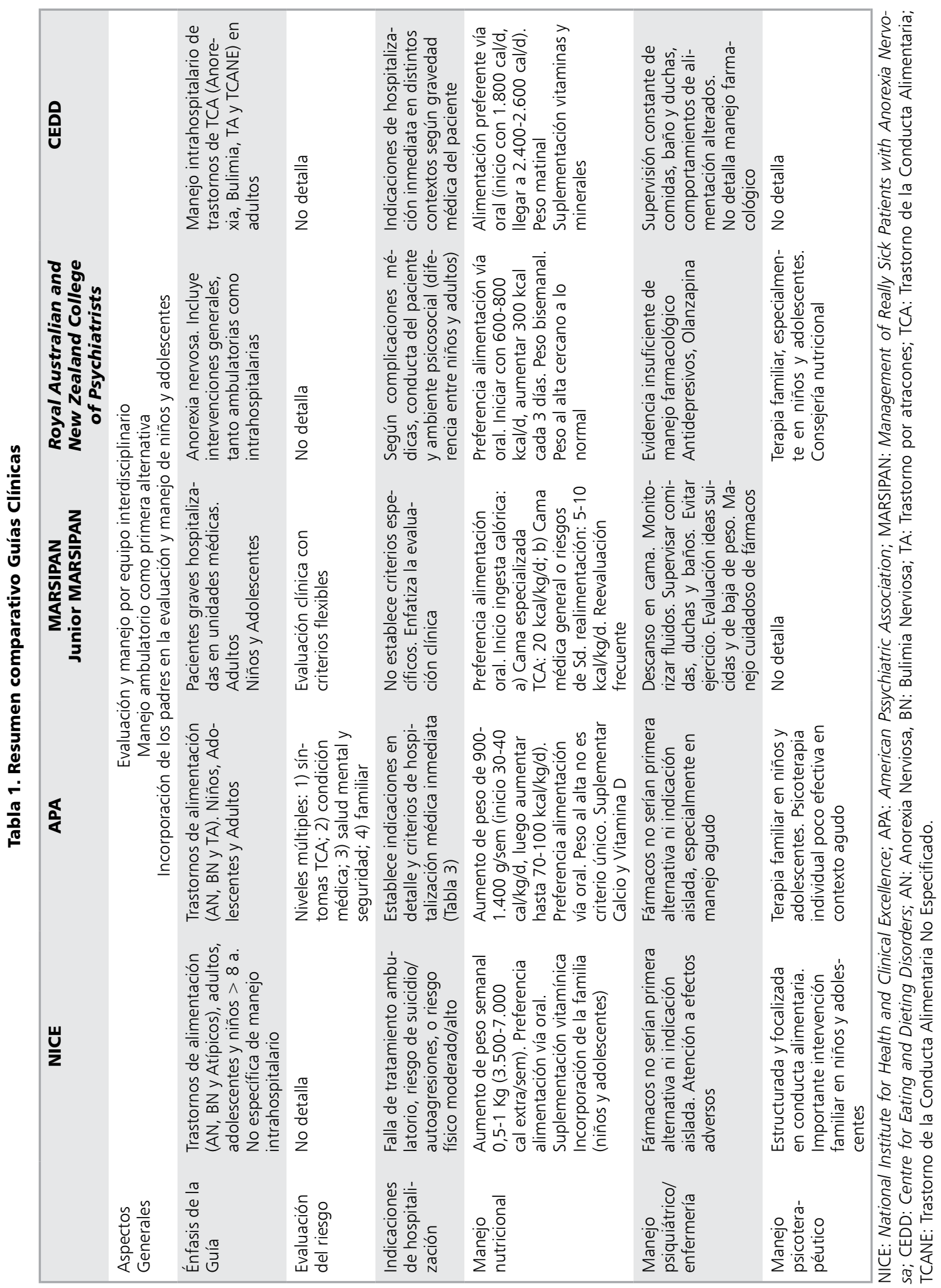


Los principales objetivos propuestos de la hospitalización en AN consisten en prevenir las complicaciones médicas secundarias a la desnutrición, manejar la comorbilidad psiquiátrica, contener el riesgo suicida si existe y alimentar en forma segura, evitando los riesgos de un síndrome de realimentación. Cada guía clínica revisada establece distintos criterios de hospitalización y, al mismo tiempo, énfasis diferentes en los procesos terapéuticos durante el período intrahospitalario. Sin embargo, hay conceptos que se repiten y que pudieran establecerse como recomendaciones generales: el énfasis en priorizar el manejo ambulatorio cuando sea posible, la importancia de la evaluación y manejo intrahospitalario por un equipo interdisciplinario y especializado que trabaje en forma coordinada, y que sostenga reuniones frecuentes -con la presencia concertada de médicos, psicólogos y nutricionistas- y la incorporación de los padres/ cuidadores en la evaluación y manejo en el caso de niños y adolescentes.

El manejo intrahospitalario de la $\mathrm{AN}$ es necesario frente a condiciones clínicas diversas que no sólo dependen del IMC, como son la velocidad y cronicidad de la pérdida de peso, el estado cardiovascular, anormalidades en el ECG, deshidratación, disminución de temperatura corporal, anormalidades bioquímicas, comportamiento en relación a la alimentación, adherencia al plan de manejo, actividad y ejercicio, riesgo de autoagresión y suicidio, comorbilidad psiquiátrica o sospecha de delirium, entre otras (Tabla 2). Las indicaciones de hospitalización son más flexibles en algunas guías que en otras; en el caso de niños y adolescentes, se destaca la evaluación del funcionamiento familiar y las posibles consecuencias sobre el desarrollo físico y psicológico de la AN en caso de cronificarse la sintomatología.

En el manejo nutricional, se privilegia la realimentación evitando suplementos alimenticios, en un contexto de acompañamiento cercano y motivante, que incorpore especialmente a los padres/ cuidadores en el caso de niños y adolescentes. En caso que la alimentación oral no sea posible, puede recurrirse a la alimentación con fórmula o al uso de SNG (diurna o aporte nocturno más alimentación convencional). La alimentación parenteral no está recomendada como manejo inicial. El inicio de la ingesta calórica plantea diferencias según el grupo de trabajo, con enfoques más conservadores en algunos casos que inician con ingesta aprox de $1.000 \mathrm{cal} /$ día y otros que inician con ingestas mayores de $1.800 \mathrm{cal} /$ día. En ambos casos recomiendan prestar atención al síndrome de realimentación y aumentar lenta y progresivamente la ingesta, además de suplementar con vitaminas y minerales.

En cuanto a las medidas conductuales durante la hospitalización, el apoyo del equipo de enfermería en la supervisión del paciente es fundamental: es necesario prestar atención al uso cotidiano de duchas y baños, evitar la actividad física exagerada o la autoagresión, además de favorecer un ambiente contenedor que promueva una conducta alimentaria normal y evite las conductas alimentarias aberrantes con el fin de disminuir la ingesta calórica.

El uso de psicofármacos no tiene un rol específico en el tratamiento intrahospitalario; su uso debe analizarse juiciosamente en cada caso de acuerdo al perfil sintomático y estado clínico de cada paciente. Por otra parte, es esperable que muchos de los síntomas psiquiátricos presentados por los pacientes respondan en forma favorable a la realimentación ${ }^{18,19}$. Particularmente, los antipsicóticos atípicos han mostrado utilidad para el manejo de la obsesividad y agitación presentes en estas pacientes $^{20}$.

La intervención familiar es habitualmente recomendada en niños y adolescentes, con evidencia creciente de que realizar este trabajo en forma precoz permitiría evitar el desarrollo de formas más persistentes de la enfermedad ${ }^{20}$. La psicoterapia individual durante la hospitalización no ha mostrado suficiente evidencia en favorecer la recuperación nutricional, aunque algunas intervenciones específicas han mostrado efectos positivos, tales como la terapia de remediación cognitiva que se asocia a una mejoría en la flexibilidad cognitiva y en la coherencia central, y el entrenamiento de habilidades sociales que mejoraría la adherencia al tratamiento intrahospitalario $^{21}$. La investigación de las intervenciones psicoterapéuticas en anorexia (la mayoría de ellas en contexto de manejo ambulatorio) se encuentra en plena expansión, aunque aún se requiere de estudios randomizados controlados de mayor tamaño y de seguimientos a más largo plazo para dar clara cuenta de su eficacia, tanto en reducir los síntomas de la enfermedad, como en mejorar la calidad de vida de las pacientes ${ }^{22}$. 
Tabla 2. Tabla comparativa de indicaciones de hospitalización en AN

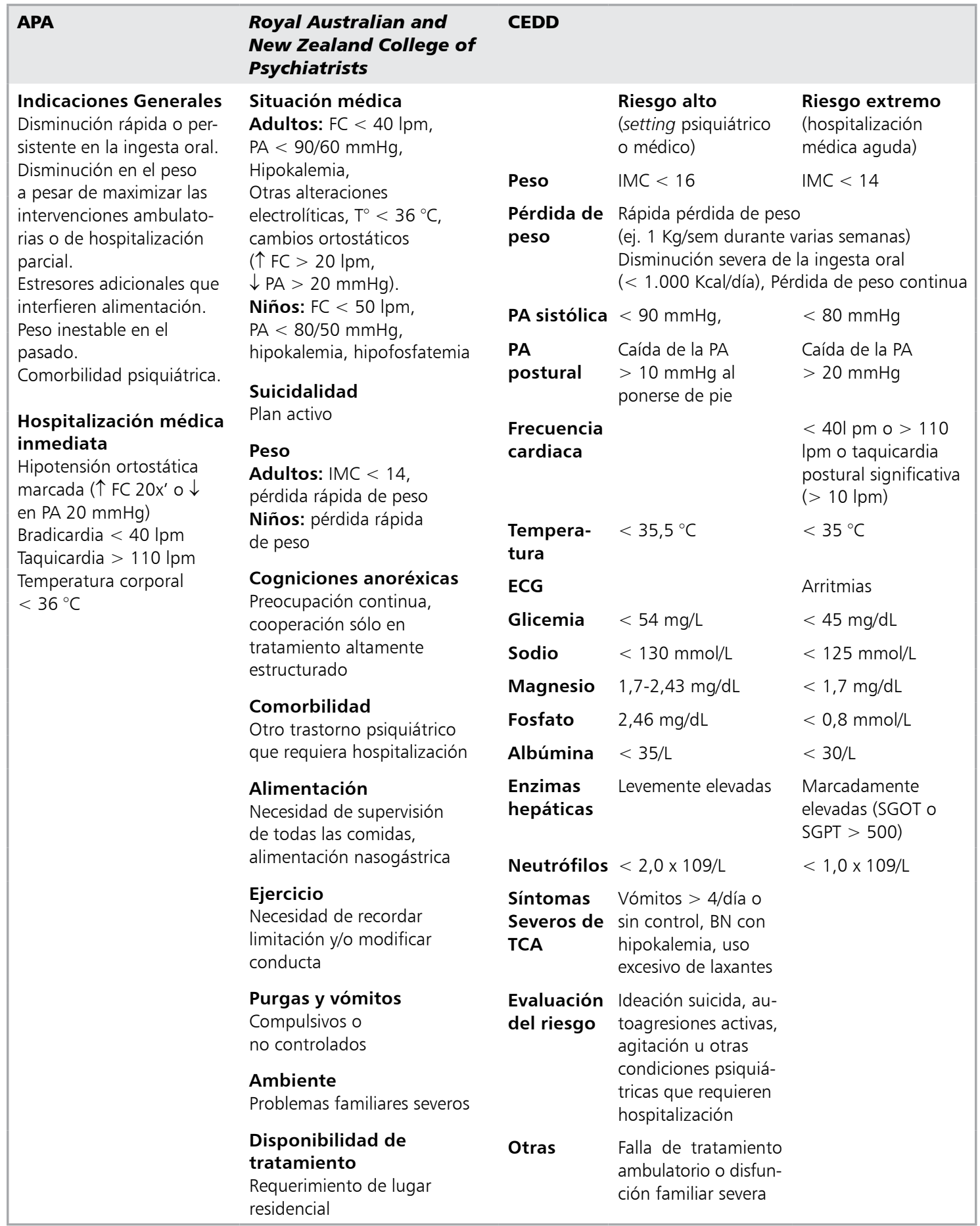

APA: American Psychiatric Association; CEDD: Centre for Eating and Dieting Disorders; FC: frecuencia cardíaca; IMC: índice de masa corporal; PA: presión arterial; Ipm: latidos por minuto; T: temperatura; SGOT: transaminasa oxalacética sérica; SGPT: transaminasa pirúvica sérica. 
Respecto a la extensión de la hospitalización, la información es escasa. Lograr la recuperación nutricional o acercarse a ésta durante el tratamiento intrahospitalario se ha relacionado con mejor evolución, en términos de menor frecuencia de recaídas y re-hospitalizaciones.

\section{Discusión}

Si bien existe consenso entre las guías sobre algunas generalidades del tratamiento de la AN, no existen propuestas de tratamiento estandarizados que ayuden a estructurar de manera más universal las decisiones clínicas frente a estos pacientes. Es destacable la escasez de guías clínicas Latinoamericanas sobre el tema, aún más de AN y su manejo más especializado a nivel intrahospitalario. Por lo mismo, no fue posible incluir alguna guía que tratara este tema en un contexto distinto al europeo o norteamericano.

La escasez y heterogeneidad de estudios clínicos randomizados y controlados, dificulta la inclusión de evidencia de mejor calidad en nuestra revisión. Si bien esto puede verse como una debilidad, las guías clínicas han sido un recurso valioso al basarse en recomendaciones de grupos de expertos y entregar información práctica. La información disponible sobre el tema no ha tenido mayores cambios en los últimos años, es por esto que algunas de estas guías no se encuentran actualizadas.

El adecuado manejo de la AN es altamente relevante considerando que se trata de una enfermedad con alto riesgo de cronificación, con una prevalencia en expansión a nivel mundial, y con evidencia científica creciente para ser considerada, junto a otros trastornos alimentarios, dentro de las principales causas de discapacidad en adolescentes. Hasta ahora los aspectos preventivos de una política de salud mental no han sido suficientemente implementados en nuestro país, como tampoco existe una adecuada cobertura para los problemas psiquiátricos más complejos, particularmente respecto a su tratamiento intrahospitalario. Una fortaleza de esta revisión es proporcionar información que sirva de referencia práctica sobre el abordaje intrahospitalario de una patología de difícil manejo y mal pronóstico, a la cual, tanto médicos especialistas, como no especialistas, se verán probablemente enfrentados. Al no existir guías nacionales al respecto, este es un primer intento de resumir la información proveniente de distintos centros dedicados por largo tiempo al manejo de estos pacientes, en virtud de que estas recomendaciones puedan ser adaptadas a nuestra realidad local, tanto en hospitales generales, como en servicios de psiquiatría.

\section{Referencias}

1. Smink F, van Hoeken D, Hoek HW. Epidemiology of Eating Disorders: Incidence, Prevalence and Mortality Rates. Curr Psychiatry Rep 2012; 14: 406-14.

2. Espie J, Eisler I. Focus on anorexia nervosa: modern psychological treatment and guidelines for the adolescent patient. Adolesc Health Med Ther 2015; 6: 9-16.

3. American Psychiatric Association. Diagnostic and statistical manual of mental disorders, DSM-5. (5th Edition). Washington, DC: American Psychiatric Association, 2013.

4. Vos T, Mathers C, Herrman H, Harvey C, Gureje O, Bui $\mathrm{D}$, et al. The burden of mental disorders in Victoria, 1996. Soc Psychiatry Psychiatr Epidemiol 2001; 36: 5362.

5. Keski-Rahkonen A, Hoek HW, Susser ES, Linna MS, Sihvola E, Raevuori A, et al. Epidemiology and course of Anorexia Nervosa in the Community. Am J Psychiatry 2007; 164: 1259-65.

6. Steinhausen $\mathrm{H}$. The outcome of anorexia nervosa in the 20th century. Am J Psychiatry 2002; 159: 1284-93.

7. Herpertz-Dahlmann B. Adolescent Eating Disorders: Update on Definitions, Symptomatology, Epidemiology, and Comorbidity. Child Adolesc Psychiatr Clin N Am 2015; 24 (1): 177-96.

8. Sullivan P. Mortality in anorexia nervosa. Am J Psychiatry 1995, 152 (7): 1073-4.

9. Arcelus J, Mitchell A, Wales J, Nielsen S. Mortality rates in patients with anorexia nervosa and other eating disorders. Arch Gen Psychiatry 2011; 68 (7): 724-31.

10. Suokas J, Suvisaari J, Gissler M, Löfman R, Linna M, Raevuori A, et al. Mortality in eating disorders: A follow-up study of adult eating disorder patients treated in tertiary care, 1995-2010. Psychiatry Res 2013; 210: 1101-6.

11. Papadopoulos F, Ekbom A, Brandt L, Ekselius L. Excess mortality, causes of death and prognostic factors in anorexia nervosa. Br J Psychiatry 2009; 194: 10-7.

12. Work Group on Eating Disorders. Practice guideline for the treatment of patients with eating disorders, Third Edition. American Psychiatric Association 2006. 
13. National Institute for Health and Clinical Excellence. Eating Disorders: Core Interventions in the Treatment and Management of Anorexia Nervosa, Bulimia Nervosa and Related Eating Disorders: Clinical Guideline 9. London, National Institute for Clinical Excellence, 2004.

14. Royal College of Psychiatrists. Junior MARSIPAN: Management of Really Sick Patients under 18 with Anorexia Nervosa. College Report 168. London, UK: Royal College of Psychiatrists 2012.

15. The Royal College of Psychiatrists, Physician and Pathologists. MARSIPAN: Management of Really Sick Patients with Anorexia Nervosa. College Report CR189. London, UK: Royal College of Psychiatrists 2014.

16. Royal Australian and New Zealand College of Psychiatrist Clinical Practice Guidelines Team for Anorexia Nervosa. Australian and New Zealand clinical practice guidelines for the treatment of anorexia nervosa. Aust N Z J Psychiatry 2004; 38: 659-70.

17. Centre for Eating and Dieting Disorders. Guidelines for the Inpatient Management of Adult Eating Disorders in General Medical and Psychiatric Settings in NSW. NSW Ministry of Health 2014.

18. Tobey JA. The Biology of Human Starvation. Am J Public Health Nations Health 1951; 41 (2): 236-7.

19. Kaye WH, Bulik CM, Thornton L, Barbarich N, Masters K. Comorbidity of anxiety disorders with anorexia and bulimia nervosa. Am J Psychiatry 2004; 161: 2215-21.

20. Fitzpatrick KK, Lock J. Anorexia nervosa. BMJ Clin Evid 2011; 2011: 1011.

21. Suárez-Pinilla $P$, Peña-Pérez C, Arbaizar-Barrenechea B, Crespo-Facorro B, Del Barrio JA, Treasure J, et al. Inpatient treatment for anorexia nervosa: a systematic review of randomized controlled trials. J Psychiatr Pract 2015; 21 (1): 49-59.

22. Hay PJ, Claudino AM, Touyz S, AbdElbaky G. Individual psychological therapy in the outpatient treatment of adults with anorexia nervosa. Cochrane Database Syst Rev 2015; (7): CD003909. 